\title{
Mesenchymal Stem Cells Modified with Short Hairpin RNA (shRNA) Constructs Targeting Insulin- like Growth Factor Binding Protein-3 (IGFBP-3) Improve Erectile Dysfunction in Old Rats
}

Xiao-Yong Pu ( $\nabla$ pxyurol@163.com )

Guangdong General Hospital https://orcid.org/0000-0002-9738-6733

Xie-WU Zhang

Guangdong Provincial People's Hospital

Xuang-Xue Zhou

Sun Yat-Sen University

Jian-Xiong Fang

Guangdong Provincial People's Hospital

Hao-Sheng Liu

Guangdong Provincial People's Hospital

Tian-Qi Liu

Guangdong Provincial People's Hospital

Hen-Jun Xiao

Sun Yat-Sen University

Xue-Cheng Bi

Guangdong Provincial People's Hospital

Dong Li

Guangdong Provincial People's Hospital

Jiu-Min Liu

Guangdong Provincial People's Hospital

\section{Research}

Keywords: Erectile dysfunction, Insulin-like growth factor binding protein-3, Mesenchymal Stem Cell, Insulin-like growth factor-1, Rats,

Posted Date: March 23rd, 2020

DOI: https://doi.org/10.21203/rs.3.rs-17848/v1 
License: (c) (i) This work is licensed under a Creative Commons Attribution 4.0 International License. Read Full License 


\section{Abstract}

Background: We have confirmed that injection of vector-based short hairpin RNA (shRNA) constructs targeting insulin-like growth factor binding protein-3 (IGFBP-3) in penis in old rat can improve erectile function. The aim of this study is to further determine the feasibility of bone marrowe derived mesenchymal stem cells (BM-MSCs) genetically modified with shRNA constructs targeting IGFBP-3 on improving erectile function in the aged rat.

Methods: 40 old (24 months) male rats were randomized divided into 4 groups: PBS-only, BM-MSC treatment and LV3-shIGFBP-3 engineered BM-MSC treatment group ( $\mathrm{n}=10$ /group). Ten 5-month-old rats were used as the young group. Four weeks after transplantation, the erectile function was assessed by cavernosal nerve stimulation. The percent of smooth muscle in corpus cavernosum was evaluated by masson's trichrome staining. IGFBP-3 expression was estimated by Western blot. The cavernous insulinlike growth factor-1 (IGF-1) and vascular endothelial growth factor (VEGF) levels were analyzed after transplantation. Apoptosis in corpus cavernosum was measured using TUNEL and the activity of nitric oxide synthase (NOS) and concentration of guanosine 3', 5'-cyclic-monophosphates (cGMP) in penile tissue were also analyzed.

Results: Four weeks after intracavernous injection, BM-MSCs alone, especially LV3-shIGFBP-3 engineered BM-MSCs treatment rats had improvements in erectile function $(p<0.05)$. The percentage of cavernosal smooth muscle was increased in BM-MSCs and LV3-shIGFBP-3 engineered BM-MSCs treatment group. IGFBP-3 protein expression was decreased obviously after injection of LV3-shIGFBP-3 engineered BMMSCs. In addition, significant lower IGFBP-3 expression was associated with increased IGF-1 expression in LV3-shIGFBP-3 engineered BM-MSCs treatment rats although higher IGF-1 and VEGF concentrations were all determined after transplantation with BM-MSCs alone or BM-MSCs modified with LV3-shIGFBP-3. Lower levels of the apoptosis were also observed in the BM-MSCs and LV3-shIGFBP-3 engineered BMMSCs treatment group. NOS activities and cGMP concentrations were also significantly increased after transplantation.

Conclusions: These findings demonstrate that BM-MSCs alone or genetically modified with LV3-shIGFBP3 can improve diminished erectile responses in the aged rat partially by increasing the smooth muscle integrity, increasing secretion of IGF-1 and VEGF, inhibiting apoptosis and increasing the NOS activity and cGMP accumulation.

\section{Background}

Erectile dysfunction (ED) is a prevailing health problem, especially in aging men [1-4]. It is estimated that 322 million men suffering from ED in 2025 [5]. 40.2\% prevalence was observed in a Chinese communitybased study in men above 40 [6]. However, the underlying mechanisms remain poorly understood especially in aging related ED. 
Insulin-like growth factor-1 (IGF-1) is a single-chain polypeptide, belonging to the insulin-like growth factor (IGF) family, which plays a key role in cellular metabolism, differentiation, proliferation, transformation and apoptosis [4]. Insulin-like growth factor binding protein-3 (IGFBP-3) is one of six IGFBPs. IGFBP-3 can regulate the binding of IGF-I with the cognate IGF-I receptor tyrosine kinase [7]. IGFBP-3 also inhibit IGF-Istimulated growth by modulating the binding of IGF-I to its receptor [7]. Furthermore, IGFBP-3 carries most of the circulating IGF-I [8]. By restricting the extravascular transit of IGF-I to the target cells, IGFBP-3 regulates the availability of IGF-I [8]. In our previous study, we have confirmed higher expression of insulin-like growth factor binding protein-3 (IGFBP-3) in old rat and the higher expression of IGFBP-3 was able to down-regulate by using the plasmid-based short hairpin RNA (shRNA) technology $[2,3]$. Intracorporeal injection of shRNA targeting IGFBP-3 was able to enhance erectile function in aged rats [2]. We also observed adenoviral gene transfer of IGF-1 or injection of IGF-1 protein to the corpus cavernosum could improve erectile function in diabetic rats and aged rats $[4,9]$.

Marrowe derived mesenchymal stem cells (BM-MSCs) have self-renewal capacity, which potential to differentiate into a wide range of cell populations. Recently, BM-MSCs were used to generate positive therapeutic effects in age-related ED and may use in clinical trial $[1,10,11]$. However, using some specific gene to modify the BM-MSCs may have the cooperative effects in treating ED and the detailed mechanisms also remain elusive. By inhibiting mRNA translation and/or inducing its degradation to silence the target gene expression, shRNA technology was used in many gene therapy related experiments. This technique has been used to ameliorate ED in aged animal models by our and others [2, 12]. In this study, based our previous serial study on IGF-I gene therapy, plasmid-based IGFBP-3 shRNA, [2, $4,9]$ we further determine whether combining BM-MSCs with IGFBP-3 shRNA technique can improve aging-related ED in rats.

\section{Methods}

\section{IGFBP-3 shRNA Overexpressing BM-MSCs}

The most validated shRNA constructs targeting IGFBP-3 was prepared as we previously described [2]. Lentiviruses expressing IGFBP-3 shRNA (LV3-shIGFBP-3) were generated by GenePharma Biotechnology Company (Shanghai, China).

Primary BM-MSCs were cultured overnight in 6-well plates $\left(1 \times 10^{4} /\right.$ well $)$ in low glucose-containing Dulbecco's modified Eagle's medium (DMEM, Gibco, Gaithersburg, MD, USA) supplemented with $20 \%$ fetal bovine serum (FBS; Gibco) at $37^{\circ} \mathrm{C}$ and $5 \% \mathrm{CO}^{2}$. The engineered BM-MSCs with LV3-shIGFBP-3 were cultured in 6-well plates at a concentration of $5 \times 10^{5} /$ well. The engineered cells were continuously cultured for 3-5 days in the incubator. Transfected engineered BM-MSCs were selected and used in vivo injection based on IGFBP-3 expression, proliferation, and other BM-MSC phenotypes (data not show).

\section{Experimental Animals}


30 old male Sprague-Dawley rats (24 months) weighting $300 \mathrm{~g}-350 \mathrm{~g}$ (Grade A) were obtained from the Animal Breeding Center of Sun Yat-Sen University and The Animal Center of Guangdong Province (Guangzhou, China). The investigation was approved by the Animal Experiment Committee of the Guangdong Provincial People's Hospital, Guangdong Academy of Medical Sciences. The old rats were randomly divided into 3 groups with ten rats each: PBS-only, BM-MSC and LV3-shIGFBP-3 engineered BMMSCs treatment group ( $n=10 /$ group). Ten 5 -month-old rats were used as the young rat group. The PBSonly rats were injected with only $200 \mu$ I PBS. The BM-MSC and LV3-shIGFBP-3 engineered BM-MSCs treatment group were treated with BM-MSCs or BM-MSC modified with LV-shIGFBP-3 by bilateral intracavernous injection under anesthesia $\left(1 \times 10^{6} \mathrm{BM}-\mathrm{MSC}\right.$ diluted in $200 \mu \mathrm{L}$ PBS$)$ as the technique we previously described [2].

\section{Measurement of Erectile Function}

As we previously technique [2-4], erectile function was assessed by measuring intracavernous pressure (ICP) following electrostimulation of the cavernous nerves after 4 weeks. The cavernosal nerve was stimulated by the electronic stimulator at a frequency of $15 \mathrm{~Hz}$ and pulse width of $30 \mathrm{~s}$ in each rat. To achieve significant erectile responses, $5 \mathrm{~V}$ was used in the current protocol. The duration of stimulation was 1 minute, with a rest period of 2-3 minutes between subsequent stimulations. Maximal ICP/MAP was recorded in each rat.

\section{Masson's Trichrome Staining}

As we previous methods [2, 4], the relative amounts of smooth muscle and collagen were measured by computer assisted histomorphometric image analysis using the Mivnt Image analysis system (Shanghai institute of optical instruments, Shanghai, China). A total of 15 fields were randomly selected and analyzed per specimen.

\section{Western Blotting}

As we previous reported [2,3], the collected corpus cavernosum tissues were homogenized in ice-cold mammalian tissue protein extraction reagent. The monoclonal anti- IGFBP-3 (1:1000 dilute) (Boster, Wuhan, China) antibody were used as the first antibody. The protein expression of IGFBP-3 relative to atublin was quantified by densitometry.

\section{Concentrations of IGF-1 and VEGF in Rat Corpus Cavernosum}

The frozen corporal tissue of each rat was also collected for IGF-1 and VEGF analysis. As the manufacturer's instructions, the IGF-1 and VEGF concentrations in rat corpus cavernosum were determined by an enzyme-linked immunosorbent assay (ELISA) kit (Shanghai Xitang Biotechnology Co., Ltd.)

\section{TUNEL Staining}


After preparation of 4- $\mu \mathrm{m}$-thick sections, the apoptotic cells in the penile tissues were evaluated by the TdT-mediated dUTP nick-end labeling (TUNEL) staining on five consecutive sections in all groups after 4 weeks using a TUNEL apoptosis detection kit (Boster, Wuhan, China), according to the manufacturer's instructions. Digital images were obtained using a Zeiss LSM 510 Meta confocal microscope (Zeiss, Oberkochen, Germany). The TUNEL picture (200x) of each group of penis tissue was analyzed and each sample was randomly selected from four fields of view. Cells with red staining were counted as apoptotic, and the ratio of apoptotic cells to the total number of cells (blue staining with DAPI) in the field of view yielded the rate of apoptosis (the apoptotic index, \%).

\section{NOS Activity and Cyclic Nucleotide Assays}

NOS activity and cGMP concentrations in penile tissues were estimated using the method as we previously reported [2].

\section{Statistical Analysis}

Data were analyzed using student's t-tests, one-way ANOVA, and $2 \times 2$ factorial ANOVA, as appropriate, to evaluate differences by SPSS version 22.0 (IBM, Armonk, NY, USA). Values were considered significant at $\mathrm{P}<0.05$. Data were expressed as mean \pm standard deviation.

\section{Results}

\section{Effect of LV3-shIGFBP-3 Engineered BM-MSCs Treatment on Erectile Responses}

The representative ICP tracing in response to electrostimulation of cavernous nerve at 4 weeks after injection is indicated in Fig. 1 (Upper). Compared with young rats, PBS-only group aged rats had significantly lower Max ICP/MAP $(p<0.05)$. At 4 weeks after the intracavernous injection of BM-MSCs and LV3-shIGFBP-3 engineered BM-MSCs, aged rats showed significantly increased Max ICP/MAP compared with the PBS-only aged group ( $p<0.05$, respectively; Fig. 1, Lower). These results showed that treatment with BM-MSCs, especially LV3-shIGFBP-3 engineered BM-MSCs, could improve ED.

\section{Masson's Trichrome Staining}

Figure 2 (Upper) shows representative histochemical results of Masson's trichrome staining. Compared with young rats, PBS-only group aged rats had significantly lower the smooth muscle contents $(p<0.05)$. After 4 weeks, when areas were quantitatively studied, the smooth muscle contents was significantly higher in the BM-MSCs and LV3-shIGFBP-3 engineered BM-MSCs treatment group $(P<0.05$, respectively, Fig. 2 Lower).

\section{Western Blot Analyses of IGFBP-3 Protein Expressions}

The IGFBP-3 protein band can be determined in cavernous tissue of all the four group rats after 4 weeks (Fig. 3 Upper). As we have observed [3] and also determined in this study, the cavernous IGFBP-3 protein 
expression was significantly higher in PBS-only aged group than in the young group rats $(P<0.05)$. Cavernous IGFBP-3 protein expression was significantly decreased after LV3-shIGFBP-3 engineered BMMSCs treatment compared with PBS-only group $(P<0.05)$. The cavernous IGFBP-3 protein levels were also decreased in BM-MSCs treatment rats compared with PBS-only group, but there is no significant difference $(P>0.05)$. It showed that the BM-MSCs alone may not obviously effect the expression of IGFBP-3 (Fig. 3 Lower).

\section{Changes of the Cavernous Concentrations of IGF-1 and VEGF in Rat Penile Tissues}

As showed in Fig. 4, after 4 weeks, compared with young rats, the concentrations of IGF-1 and VEGF were significantly decreased in PBS-only aged rat penile tissues, which were confirmed by ELISA $(p<0.05$, respectively). The intracavernous administration of BM-MSCs and LV3-shIGFBP-3 engineered BM-MSCs increased cavernous IGF- 1 and VEGF concentrations compared with PBS-only aged rats $(p<0.05$, respectively). Moreover, the higher cavernous IGF-1 concentrations were determined after LV3-shIGFBP-3 engineered BM-MSCs treatment than in BM-MSCs only treatment $(p<0.05)$. However, no significant higher expression of the cavernous VEGF concentrations were observed after LV3-shIGFBP-3 engineered BM-MSCs treatment than in BM-MSCs only treatment $(p>0.05)$.

\section{Assessment of Apoptotic Index TUNEL Staining}

In order to determine whether BM-MSCs or LV3-shIGFBP-3 engineered BM-MSCs injection could reduce apoptosis, a TUNEL assay was carried out to detect apoptotic cells in all groups (Fig. 5, Upper). The apoptotic index (\%) of the PBS-only aged group was significantly lower than the young group $(P<0.05$, Fig. 5, Lower). The obviously lower apoptotic index (\%) was determined in BM-MSCs treatment group and LV3-shIGFBP-3 engineered BM-MSCs treatment group than in PBS-only group $(P<0.05$, respectively, Fig. 5, Lower),suggesting that BM-MSCs alone or BM-MSCs modified with LV3-shIGFBP-3 triggers an decrease in apoptotic cells. It showed that BM-MSCs alone or BM-MSCs modified with IGFBP-3 shRNA injection had a potentially beneficial effect on suppressing apoptosis and increasing the ED.

\section{Changes of NOS Activity and of cGMP Concentration}

The calcium-dependent NOS activity and the CGMP concentrations in young rats were significantly higher than in the PBS-only group $(P<0.05$, respectively, Fig. 6$)$. After 4 weeks treatment, the NOS activity and the cGMP concentrations were all increased respectively in the BM-MSCs and LV3-shIGFBP-3 engineered BM-MSCs group $(P<0.05$, respectively, Fig. 6).

\section{Discussion}

This study reveals that transplantation of BM-MSCs alone or BM-MSCs modified with virus-based shRNA against IGFBP-3 improved ED in aged rats. The BM-MSCs and the shRNA against IGFBP-3 may have the cooperative effects in increasing ED. Furthermore, the intracavernous administration of BM-MSCs alone or BM-MSCs modified with shRNA against IGFBP-3 increased the concentrations of IGF-1 and VEGF in 
penile tissues. The BM-MSCs modified with shRNA against IGFBP-3 significantly increased the concentrations of cavernous IGF-1, which indicate the BM-MSCs and shRNA against IGFBP-3 may have the cooperative effects in changing the IGF-1 level. Moreover, the erectile responses increased by BMMSCs alone or BM-MSCs modified with shRNA against IGFBP-3 might have been caused by decreased the cell apoptosis, increased the smooth muscle integrity and NOS activity and accumulated concentration of cGMP. In this study, we did not design the plasmid shRNA against IGFBP-3 as the control group because we have confirmed that single injection of vector-based shRNA against IGFBP-3 can alleviate erectile function in aged rats in our previous study[2]. Thus, the transplantation of BM-MSCs modified with shRNA against IGFBP-3 appeared to be another effective treatment in aging related rats.

We have observed the higher expression of mRNA and protein of IGFBP-3 in old rat penile tissues which is related ED [3]. The higher expressions of IGFBP-3 were also observed in corpus cavernosum tissues of DM rats [13] and hypertensive rats [14]. It showed that IGFBP-3 might play an vital role in the development of ED. IGFBP-3 was produced largely by the liver, which is a 40- to 45-kDa glycoprotein, was the major IGFBP present in serum [15]. The growth of human intestinal smooth muscle cells can be inhibited by secretion of IGFBP-3 [16]. IGFBP-3 also acts to inhibit IGF-I-stimulated cell growth [17]. By restricting the extravascular transit of IGF-I to the target cells, IGFBP-3 regulates the availability of IGF-I and carries most of the circulating IGF-I [8]. We have confirmed that plasmid-based shRNA against IGFBP3 alleviate age related ED in the rat [2]. In this study, we further observed inhibiting the IGFBP-3 by virusbased shRNA combined with BM-MSCs can improve the cavernous IGF-1 concentrations. It showed inhibiting the IGFBP-3 and improving the IGF-1 are all contributed to alleviate age related ED. Zhou. et al. [18-20]recently observed that plasmid-based shRNA targeting IGFBP-3 improve diabetic related ED in the rat by effecting the testosterone concentrations and rehabilitating the dyslipidaemia.

Transplantation of mesenchymal stem cells (MSCs) to treat ED is a promising new therapeutic approach. Depending on the different stimuli or signals, MSCs can differentiation into various specialized cell types, such as smooth muscles, which is benefit for correcting ED [21]. Until now, several types of MSCs, such as adipose tissue-derived stem cells (ADSCs), urinary stem cells (USCs) and BM-MSC are capable of differentiation into endothelial cells, or cavernous smooth muscle cells. This unique character and ability of BM-MSCs has been used to treat ED in rat even in clinical trial $[22,23]$. Treating of ED by BM-MSCs is still only in the clinic trial phase. However, the primary clinical promising results are very exciting. Yiou et al. [23] evaluated the safety of transplantation of BM-MSCs for postradical prostatectomy ED. Twelve patients with localized prostate cancer and ED were enrolled the study. Greater improvement in spontaneous erections was observed and no serious side effects were occurred after 1 year followup. Recently, an open label phase I clinical trial was also conducted to evaluate the safety and potential therapeutic effect of two intracavernous autologous BM-MSCs injections in diabetic patients with ED [22]. Four diabetic patients were included in the study and proved that intracavernous autologous BM-MSC injections were tolerability, safety and efficacy. More clinical trial with more samples is urgent to elucidate the important role of BM-MSCs in treating ED. 
Apply genetically modified stem cells in treatment of ED may be another good choice in the future depending on the cooperative effects. It is showed that combing cell therapy with gene therapy remarkably improve differentiation of implanted MSCs, revascularization, and innervation in genitourinary tissues, especially in the treatment of ED.[24] Bivalacqua. et al. [11] first used the genetic BM-MSCs modification technique on treating ED, which using MSCs modified with endothelial nitric oxide synthase (eNOS) to reverse age-associated ED. Recently, Liu. et al. [1] evaluated the therapeutic efficiency of mcroRNA-145 engineered BM-MSCs on alleviating ED in aged rats. Jeon. et al. [25] observed the beneficial effects of stromal cell-derived factor-1 (SDF-1) engineered BM-MSCs on improving streptozotocin (STZ)-induced DM ED rat model. He. et al. [26] assessed the positive therapeutic effects of the BM-MSCs modified with KCNMA1 on the improvement of erectile function in diabetes-associated ED. Qiu. et al. [27] investigated the effects of combined strategy of MSCs injection with vascular endothelial growth factor (VEGF) 164 gene therapy for the treatment of diabetic rats. In this study, we also confirmed BM-MSCs alone or BM-MSCs modified with shRNA targeting IGFBP-3 effectively reversing age-associated ED. The BM-MSCs and the virus-based shRNA may have the cooperative effects in treating ED due to aging.

The mechanism of transplantation BM-MSCs on treatment of ED remains controversial. Producing some bioactivity factors may contribute to the therapeutic effects. A paracrine action rather than the differentiation of stems cells may play a major role in the efficacy of stem cell transplanltion [28]. This study revealed that the intracavernous administration of BM-MSCs alone or BM-MSCs modified with shRNA against IGFBP-3 increased the cavernous concentrations of IGF-1 and VEGF in aged rats. Thus, the transplantation of BM-MSCs alone or engineered BM-MSCs appeared to be an effective treatment for improving aged ED through stimulating the secretion of IGF-1 and VEGF. IGF-1 is a single-chain polypeptide and may play a vital role in penile erection. We have confirmed that gene transfer of IGF-1 and injection of IGF-1 protein improved erectile function in diabetic rats and aged rats $[4,9]$. In this study, we observed that LV3-shIGFBP-3 engineered BM-MSCs have more effect in improving concentration of cavernous IGF-1 than BM-MSCs only, which indicated that inhibiting the IGFBP-3 and BM-MSCs have the cooperative effects on improving concentration of IGF-1. VEGF is a chemical signal protein, which play an important role in vasculogenesis and angiogenesis, especially in ED. The lower expression of VEGF in the erectile tissues of aged rats has been confirmed. Moreover, gene transfer of VEGF alone or BM-MSCs modified with VEGF have been observed in improving erectile function in diabetes and age related animal models $[27,29]$. Furthermore, Yang. et al.[28] revealed that ADSCs can improve erectile function through the secretion of cavernous IGF-1, bFGF, and VEGF in old rats. This study indicated that BM-MSCs alone, especially BM-MSCs modified with shRNA targeting IGFBP-3 treatment can improve aging-related ED partially owing to the secretion of IGF-1 and VEGF.

Apoptosis of cavernosal cells has been confirmed in some of experimental models of ED, especially in diabetes rats and aged rats. It is generally assumed that the smooth muscle cells apoptosis is the important reason in the development of ED. The smooth muscle and endothelial cells of the cavernosal arteries apoptosis were also observed after bilateral cavernous nerve ablation (BCNA) [30]. In this series, BM-MSCs alone or BM-MSCs modified with IGFBP-3 shRNA triggers an increase in apoptotic cells. It 
showed that BM-MSCs had a potentially beneficial effect on suppressing apoptosis and the BM-MSCs modified with shRNA more prominent. It also showed massive cell apoptosis may play a vital role in the pathophysiology of postprostatectomy ED [30]. Fall. et al. [30] determined, in postprostatectomy ED, BMMSCs improved erectile function by inhibiting apoptosis. Liu. et al. [31] revealed that ADSCs modified with hepatocyte growth factor (HGF) improved erectile function accompanying lower cell apoptotic index in corpus cavernosum in diabetic rats. All these studies showed that intracavernosal transplantation of MSCs protected against cell apoptosis and improved the erectile function. Our results also showed that BM-MSCs alone or BM-MSCs modified with IGFBP-3 shRNA were effective for maintaining the amount of smooth muscle, improving the NOS activity and cGMP concentrations. Therefore, we suggest that the major mechanisms underlying the effects of BM-MSCs alone or BM-MSCs modified with IGFBP-3 shRNA in improving the erectile response are may also relate to the preservation of the intergrity of corporeal smooth muscles and the NO-cGMP signaling pathways in aged rats [2].

Although the initial result is promising, future studies should determine the long-term therapeutic effects after administration in 3 months, even in 6 months. It is limited that we only focused on the secretion of cavernous IGF-1 and VEGF for the vital roles of IGF-1 and VEGF in ED. Because of the systemic nature of IGFBP-3, the impact of IGFBP-3 combined with BM-MSCs on other vascular and targets, such as kidney, liver, spleen, aorta etc, also should be determined in the future. Combining administration IGF-1 with IGFBP-3 shRNA, even with BM-MSCs may produce maximum cooperative effects will be performed in our future studies.

\section{Conclusions}

This study showed that BM-MSCs alone can significantly improve erectile function in aged rats, but in combination with virus-based shRNA targeting IGFBP-3 the improvement was more prominent. The possible mechanism may contribute to the higher content of smooth muscle, higher activity of NOS and cGMP accumulation and lower cell apoptotic index in penile tissues. Combination of the BM-MSCs with shRNA targeting IGFBP-3 may have the cooperative in improving the erectile function in aged rats, and this effect might be closely related to the increasing the secretion of cavernous IGF-1 and download the IGFBP-3.

\section{Declarations}

Acknowledgements

We thank all of the members in the lab. We are grateful to the financial support of the National Natural Science Foundation and the Guangdong Provincial Science Foundation.

Authors' contributions

XYP designed and performed the experiments, analyzed the data, and wrote the manuscript. XWZ, JXF, TQL carried out the experiments. XXZ, XCB and DL performed some of the research. XXZ and HJX helped 
to review the manuscript. JML participated in the experimental design, provided financial support, and supervised the manuscript. All authors read and approved the final manuscript.

Funding This work was financially supported by National Natural Science Foundation of China (81570691,81270855), Guangdong Provincial Natural Science Foundation (2019A1515012019, 2017A030313715), Science and technology program of Guangdong Province (2017ZC0275) \The open project of Key Laboratory of Male Reproduction and Genetics, National Health and Family Planning Commission (KF201801).

Availability of data and materials

For data availability, please contact the corresponding author.

Ethics approval and consent to participate

All experimental protocols were approved by the Animal Experiment Committee of the Guangdong Provincial People's Hospital, Guangdong Academy of Medical Sciences.

Consent for publication

Not applicable

Competing interests

The authors declare that they have no competing interests.

\section{Abbreviations}

IGFBP-3:Insulin-like growth factor binding protein-3; shRNA = Short hairpin RNA; BM-MSCs:Bone marrowe derived mesenchymal stem cells; ED: Erectile dysfunction; ICP:intracavernosal pressure; IGF-1: Insulin-like growth factor-1; VEGF: Vascular endothelial growth factor; NOS: nitric oxide synthase; cGMP:guanosine 3', 5'-cyclic-monophosphates; eNOS:endothelial nitric oxide synthase.

\section{References}

1.

Liu Q, Cui Y, Lin H, Hu D, Qi T, Wang B, Huang Z, Chen J, Li K, Xiao H. MicroRNA-145 engineered bone marrow-derived mesenchymal stem cells alleviated erectile dysfunction in aged rats. Stem Cell Res Ther. 2019;10(1):398.

2.

Pu XY, Wen AM, Liu JM, Zheng XG, Xiao HJ, Xu ZP, Wang HP, Li D, Zhang RL. shRNA constructs targeting IGFBP-3 alleviate age related erectile dysfunction in the rat. J Urol. 2014;192(3):990-6.

3. 
Pu XY, Zheng XG, Zhang Y, Xiao HJ, Xu ZP, Liu JM, Wang HP, Wen AM, Zhou XX, Wu YL. Higher expression of mRNA and protein of insulin-like growth factor binding protein-3 in old rat penile tissues: implications for erectile dysfunction. J Sex Med. 2011;8(8):2181-90.

4.

Pu XY, Wang XH, Gao WC, Yang ZH, Li SL, Wang HP, Wu YL. Insulin-like growth factor-1 restores erectile function in aged rats: modulation the integrity of smooth muscle and nitric oxide-cyclic guanosine monophosphate signaling activity. J Sex Med. 2008;5(6):1345-54.

5 .

Ayta IA, McKinlay JB, Krane RJ. The likely worldwide increase in erectile dysfunction between 1995 and 2025 and some possible policy consequences. BJU Int. 1999;84(1):50-6.

6.

Bai Q, Xu QQ, Jiang H, Zhang WL, Wang XH, Zhu JC. Prevalence and risk factors of erectile dysfunction in three cities of China: a community-based study. Asian J Androl. 2004;6(4):343-8.

7.

Kelley KM, Oh Y, Gargosky SE, Gucev Z, Matsumoto T, Hwa V, Ng L, Simpson DM, Rosenfeld RG. Insulinlike growth factor-binding proteins (IGFBPs) and their regulatory dynamics. Int J Biochem Cell Biol. $1996 ; 28(6): 619-37$.

8.

Vorwerk P, Oh Y, Lee PD, Khare A, Rosenfeld RG. Synthesis of IGFBP-3 fragments in a baculovirus system and characterization of monoclonal anti-IGFBP-3 antibodies. J Clin Endocrinol Metab. 1997;82(7):236870.

9.

Pu XY, Hu LQ, Wang HP, Luo YX, Wang XH. Improvement in erectile dysfunction after insulin-like growth factor-1 gene therapy in diabetic rats. Asian J Androl. 2007;9(1):83-91.

10.

Abdel Aziz MT, El-Haggar S, Mostafa T, Atta H, Fouad H, Mahfouz S, Rashed L, Sabry D, Senbel A, Ali GA. Effect of mesenchymal stem cell penile transplantation on erectile signaling of aged rats. Andrologia. 2010;42(3):187-92.

11.

Bivalacqua TJ, Deng W, Kendirci M, Usta MF, Robinson C, Taylor BK, Murthy SN, Champion HC, Hellstrom WJ, Kadowitz PJ. Mesenchymal stem cells alone or ex vivo gene modified with endothelial nitric oxide synthase reverse age-associated erectile dysfunction. Am J Physiol Heart Circ Physiol. 2007;292(3):H1278-90.

12.

Magee TR, Kovanecz I, Davila HH, Ferrini MG, Cantini L, Vernet D, Zuniga FI, Rajfer J, Gonzalez-Cadavid NF. Antisense and short hairpin RNA (shRNA) constructs targeting PIN (Protein Inhibitor of NOS) ameliorate aging-related erectile dysfunction in the rat. J Sex Med. 2007;4(3):633-43.

13.

Soh J, Katsuyama M, Ushijima S, Mizutani Y, Kawauchi A, Yabe-Nishimura C, Miki T. Localization of increased insulin-like growth factor binding protein-3 in diabetic rat penis: implications for erectile 
dysfunction. Urology. 2007;70(5):1019-23.

14.

Zhou ZY, Yang ZH, Wang XH, Cao H, Chen D, Wang YZ, Zhou HH, Peng M, Liu QL, Wan SP. Increased expression of insulin-like growth factor-binding protein-3 is implicated in erectile dysfunction in twokidney one-clip hypertensive rats after propranolol treatment. Asian J Androl. 2011;13(6):851-5. 15.

Hochscheid R, Jaques G, Wegmann B. Transfection of human insulin-like growth factor-binding protein 3 gene inhibits cell growth and tumorigenicity: a cell culture model for lung cancer. J Endocrinol. 2000;166(3):553-63.

16.

Kuemmerle JF, Murthy KS, Bowers JG. IGFBP-3 activates TGF-beta receptors and directly inhibits growth in human intestinal smooth muscle cells. Am J Physiol Gastrointest Liver Physiol. 2004;287(4):G795802.

17.

Bushman TL, Kuemmerle JF. IGFBP-3 and IGFBP-5 production by human intestinal muscle: reciprocal regulation by endogenous TGF-beta1. Am J Physiol. 1998;275(6 Pt 1):G1282-90.

18.

Zhou ZY, Li F, Cheng SP, Huang H, Peng BW, Wang J, Liu CM, Xing C, Sun YL, Bsoul N, Pan H, Yi CJ, Liu $\mathrm{RH}$, Zhong GJ. Short hairpin ribonucleic acid constructs targeting insulin-like growth factor binding protein-3 rehabilitated decreased testosterone concentrations in diabetic rats. Med Sci Monit. 2015;21:94-9.

19.

Zhou ZY, Cheng SP, Huang H, Wang J, Pan H, Liu CM, Xing C, Sun YL, Liu RH, Zhong GJ. Short hairpin ribonucleic acid constructs targeting insulin-like growth factor binding protein-3 rehabilitated dyslipidaemia in diabetic rats. Andrologia. 2016;48(1):59-64.

20.

Zhou ZY, Zhong GJ, Cheng SP, Huang H, Wang J, Pan H, Liu CM, Xing C, Sun YL, Liu RH, Li F. Short hairpin rna targeting insulin-like growth fator binding protein-3 restores the bioavailability of insulin-like growth factor-1 in diabetic rats. Int Braz J Urol. 2016;42(1):139-45.

21.

Zhang H, Albersen M, Jin X, Lin G. Stem cells: novel players in the treatment of erectile dysfunction. Asian J Androl. 2012;14(1):145-55.

22.

Al Demour S, Jafar H, Adwan S, AlSharif A, Alhawari H, Alrabadi A, Zayed A, Jaradat A, Awidi A. Safety and Potential Therapeutic Effect of Two Intracavernous Autologous Bone Marrow Derived Mesenchymal Stem Cells injections in Diabetic Patients with Erectile Dysfunction: An Open Label Phase I Clinical Trial. Urol Int. 2018;101(3):358-65.

23.

Yiou R, Hamidou L, Birebent B, Bitari D, Lecorvoisier P, Contremoulins I, Khodari M, Rodriguez AM, Augustin D, Roudot-Thoraval F, de la Taille A, Rouard H. Safety of Intracavernous Bone Marrow- 
Mononuclear Cells for Postradical Prostatectomy Erectile Dysfunction: An Open Dose-Escalation Pilot Study. Eur Urol. 2016;69(6):988-91.

24.

Li L, Zhang D, Li P, Damaser M, Zhang Y. Virus integration and genome influence in approaches to stem cell based therapy for andro-urology. Adv Drug Deliv Rev. 2015;82-83:12-21.

25.

Jeon SH, Zhu GQ, Bae WJ, Choi SW, Jeong HC, Cho HJ, Ha US, Hong SH, Lee JY, Kwon EB, Kim HJ, Lee SM, Kim HY, Kim SW. Engineered Mesenchymal Stem Cells Expressing Stromal Cell-derived Factor-1 Improve Erectile Dysfunction in Streptozotocin-Induced Diabetic Rats. Int J Mol Sci, 2018;19(12). 26.

He Y, He W, Qin G, Luo J, Xiao M. Transplantation KCNMA1 modified bone marrow-mesenchymal stem cell therapy for diabetes mellitus-induced erectile dysfunction. Andrologia. 2014;46(5):479-86.

27.

Qiu X, Sun C, Yu W, Lin H, Sun Z, Chen Y, Wang R, Dai Y. Combined strategy of mesenchymal stem cell injection with vascular endothelial growth factor gene therapy for the treatment of diabetes-associated erectile dysfunction. J Androl. 2012;33(1):37-44.

28.

Yang J, Zhang Y, Zang G, Wang T, Yu Z, Wang S, Tang Z, Liu J. Adipose-derived stem cells improve erectile function partially through the secretion of IGF-1, bFGF, and VEGF in aged rats. Andrology. 2018;6(3):498-509.

29.

Park K, Ahn KY, Kim MK, Lee SE, Kang TW, Ryu SB. Intracavernosal injection of vascular endothelial growth factor improves erectile function in aged rats. Eur Urol. 2004;46(3):403-7.

30.

Fall PA, Izikki M, Tu L, Swieb S, Giuliano F, Bernabe J, Souktani R, Abbou C, Adnot S, Eddahibi S, Yiou R. Apoptosis and effects of intracavernous bone marrow cell injection in a rat model of postprostatectomy erectile dysfunction. Eur Urol. 2009;56(4):716-25.

31.

Liu T, Peng Y, Jia C, Fang X, Li J, Zhong W. Hepatocyte growth factor-modified adipose tissue-derived stem cells improve erectile function in streptozotocin-induced diabetic rats. Growth Factors.

$2015 ; 33(4): 282-9$.

\section{Figures}



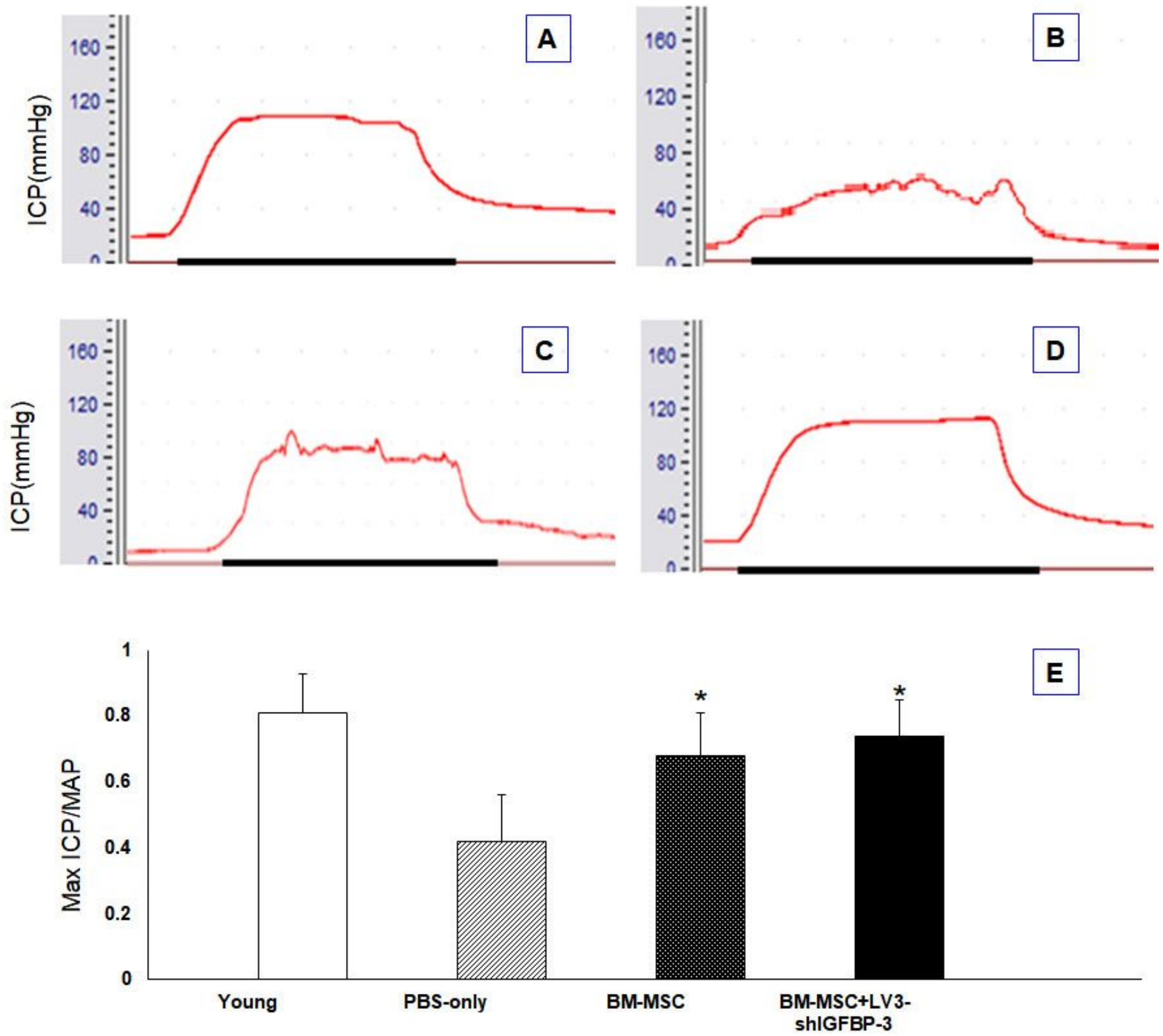

Figure 1

Comparison of erectile function among groups. Results in 10 rats per group. Upper: Representative ICP responses to cavernous nerve electrical stimulation in the young group (A), PBS-only (B), BM-MSCs treatment group (C) and the LV3-shIGFBP-3 engineered BM-MSCs treatment group (D) at 4 weeks. Bold line indicates 1 minute of electrical stimulation to cavernous nerve. Lower: ICP/MAP data were summarized in the young group, PBS-only group, the BM-MSCs treatment group and the LV3-shIGFBP-3 engineered BM-MSCs treatment group. Single asterisk indicates significantly different vs the PBS-only group $(\mathrm{p}<0.05$, respectively). 

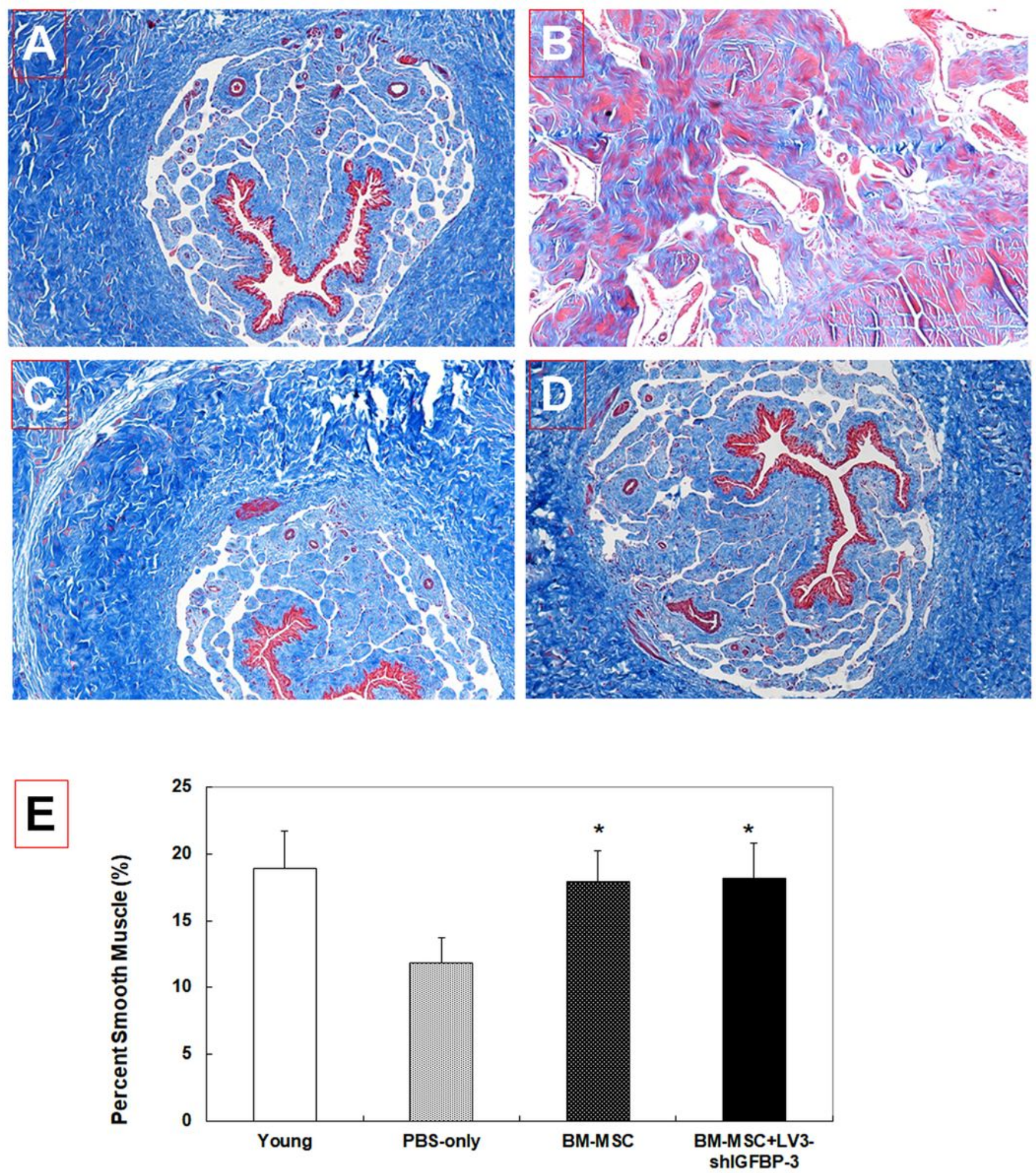

Figure 2

Representative images of Masson Trichrome staining in the corpus cavernosum. Results in 10 rats per group. Upper: Representative histochemical results of Masson's trichrome staining $(\times 100)$ in the young group (A), PBS-only (B), BM-MSCs treatment group (C) and the LV3-shIGFBP-3 engineered BM-MSCs treatment group (D) at 4 weeks. Lower: Volumetric densities of corpus cavernosum smooth muscle data 
were summarized in 4 groups. Single asterisk indicates significantly different vs the PBS-only group $(p<0.05$, respectively).

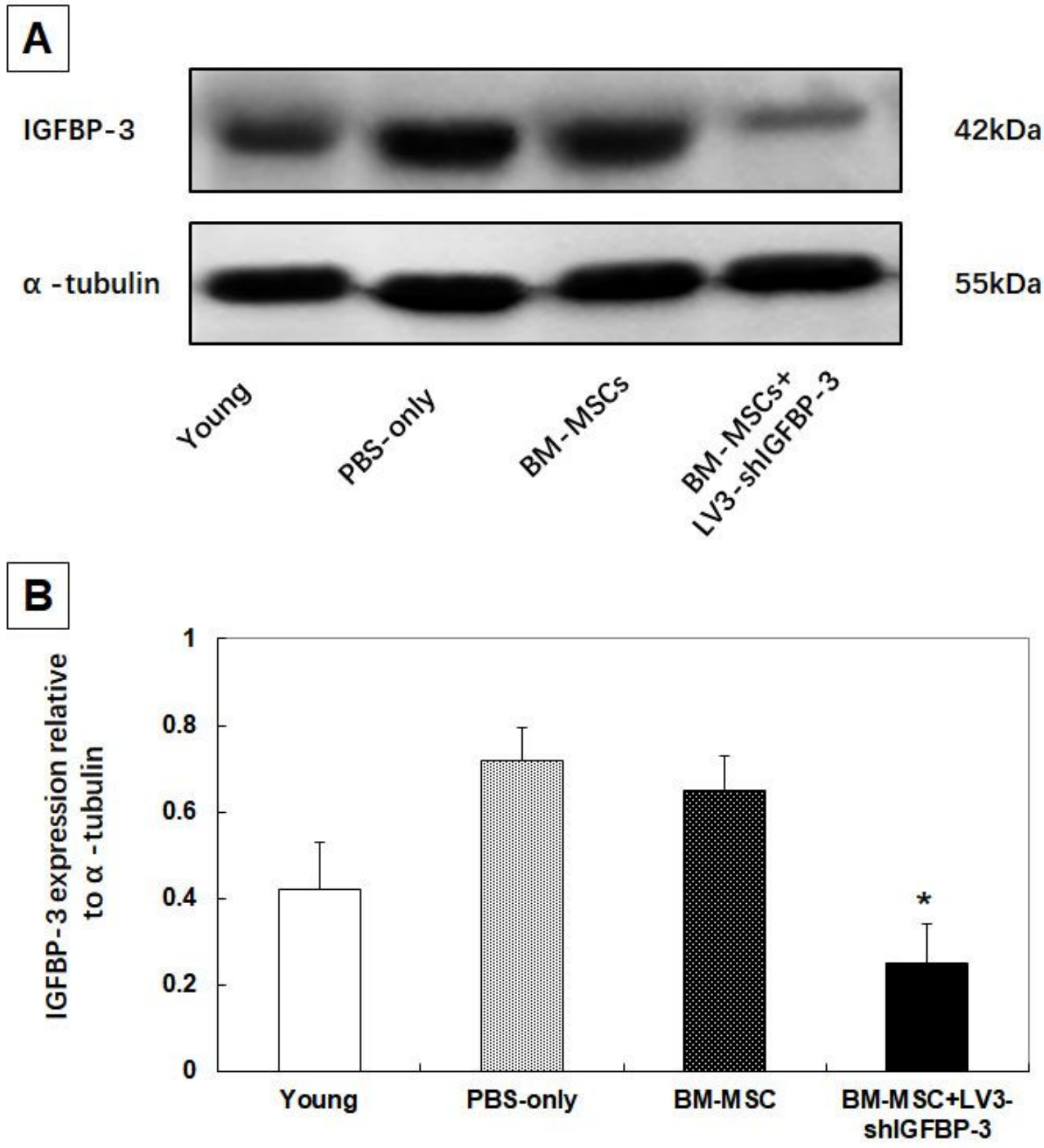

Figure 3

Western blotting quantitative analysis for IGFBP-3 protein. Results in 10 rats per group. Upper:

Representative IGFBP-3 protein expression in penile carvernous tissue according to western blot in the young group, PBS-only, BM-MSCs treatment group and the LV3-shIGFBP-3 engineered BM-MSCs treatment group at 4 weeks. Lower: The relative expression of IGFBP-3 protein in penile carvernous tissue in the 4 groups. The Single asterisk indicates significantly different vs the PBS-only group and the BMMSCs group ( $<<0.05$, respectively). 

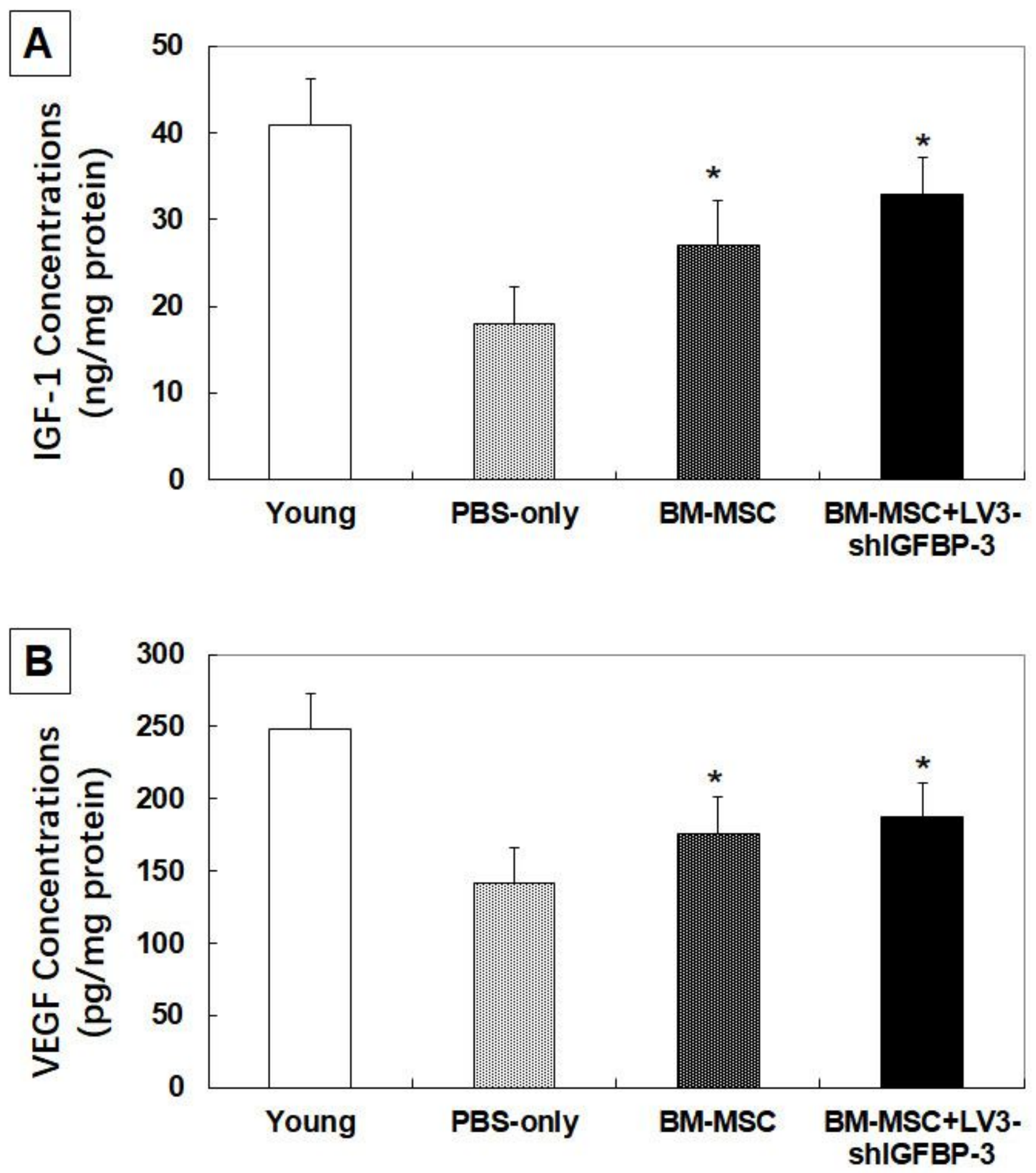

Figure 4

The changes of the IGF-1 and VEGF concentrations in corpus cavernosum. Results in 10 rats per group. Upper: Cavernous IGF-1 concentrations were determined by ELISA in the young group, PBS-only, BM-MSCs treatment group and the LV3-shIGFBP-3 engineered BM-MSCs treatment group at 4 weeks. Lower: Cavernous VEGF concentrations were determined by ELISA in the 4 groups. The single asterisk $\left(^{*}\right)$ indicates significantly different vs the PBS-only group ( $p<0.05$, respectively). The hash (\#) indicates 
significantly different vs the BM-MSCs treatment group $(p<0.05)$. The ampersand $(\&)$ indicates no significantly different vs the BM-MSCs treatment group $(p>0.05)$.
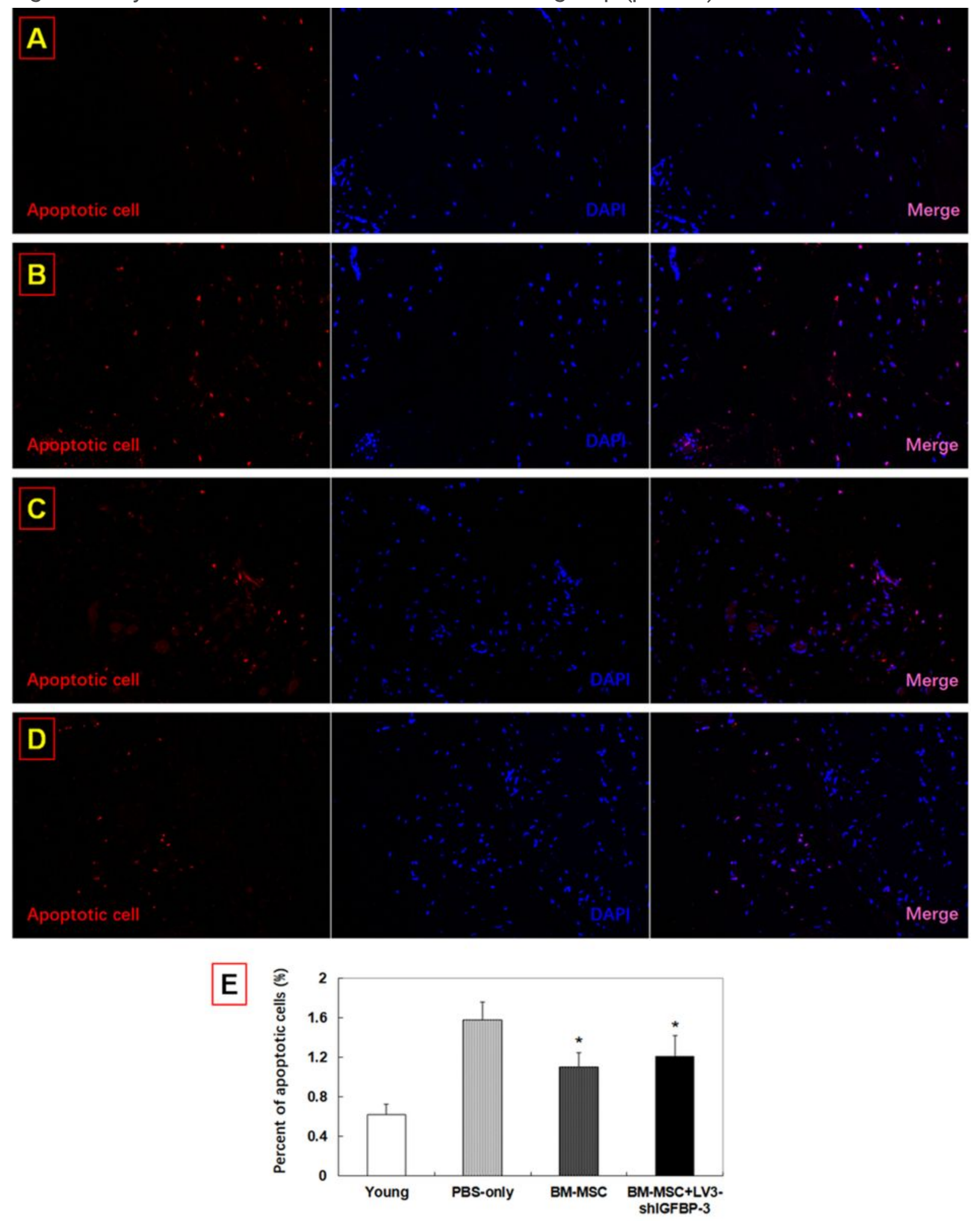

\section{Figure 5}

Representative TUNEL staining map and comparison of apoptotic index in the corpus cavernosum of each group. Results in 10 rats per group. Upper: Representative TUNEL staining $(\times 200)$ in the young group (A), PBS-only (B), BM-MSCs treatment group (C) and the LV3-shIGFBP-3 engineered BM-MSCs treatment 
group (D) at 4 weeks. Positive apoptotic cell nucleus is fluorescent red. Lower: The data of the percent of apoptotic cells (apoptosis index) were summarized in 4 groups. Single asterisk indicates significantly different vs the PBS-only group ( $p<0.05$, respectively).
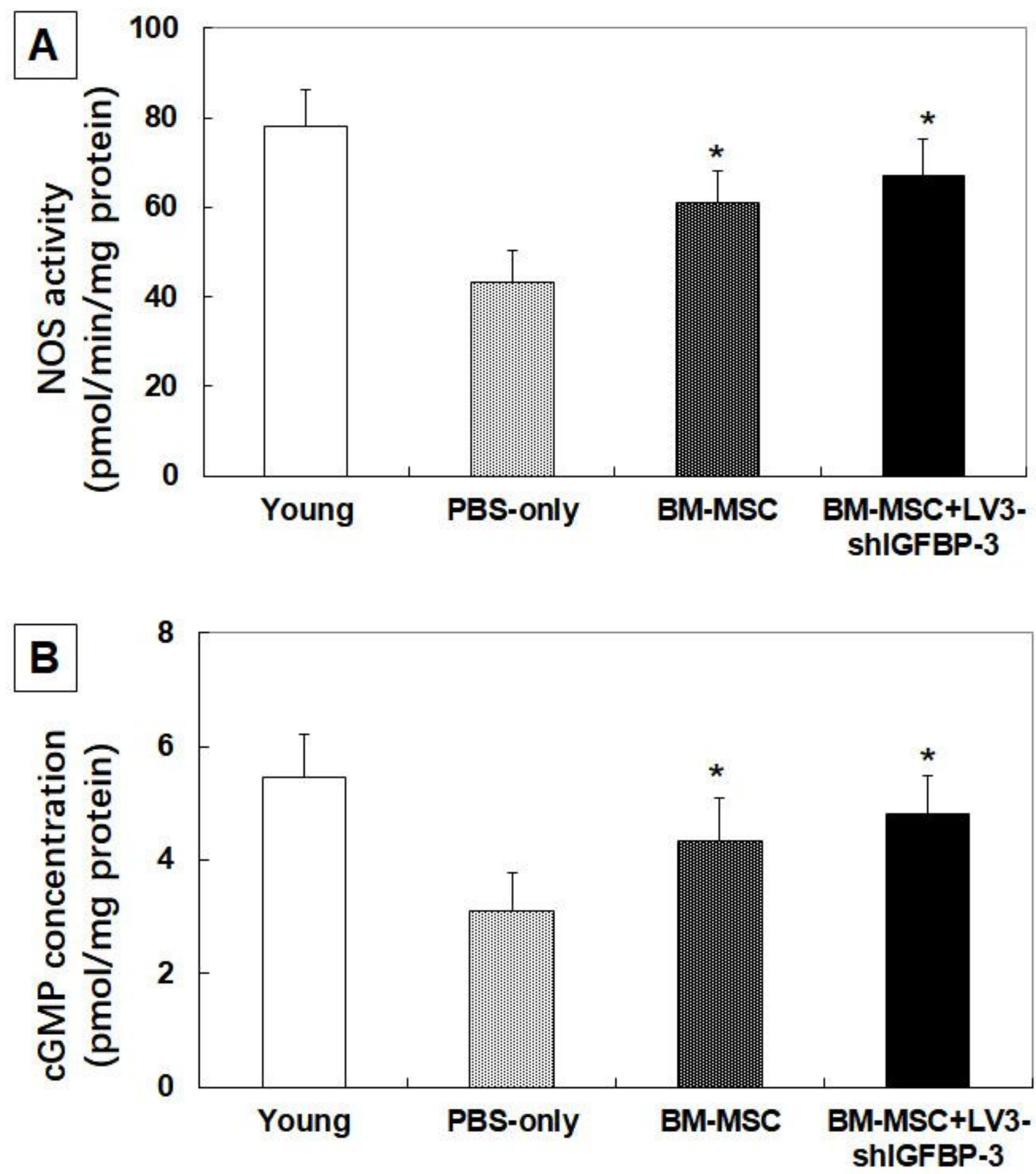

Figure 6

Changes of the NOS activity and CGMP concentration. Results in 10 rats per group. Upper: NOS activity determined by hemoglobin oxidation assay in the young group, PBS-only, BM-MSCs and the LV3- 
shIGFBP-3 engineered BM-MSCs treatment groupat 4 weeks. Lower: cGMP concentration determined by ELISA in the penile tissues in the 4 groups. Single asterisk indicates significantly different vs the PBS-only group ( $p<0.05$, respectively). 